\title{
Analytical and micromagnetic study of a Néel domain wall
}

\author{
K. Rivkin, K. Romanov, Ar. Abanov, Y. Adamov, and W. M. Saslow \\ Department of Physics, Texas A \&M University, College Station, Texas 77843, USA
}

(Received 15 July 2007; published 13 June 2008)

\begin{abstract}
For ferromagnets with exchange, dipolar interaction, and uniaxial anisotropy, by both analytic methods and micromagnetic simulations we study Néel domain walls in thin ferromagnetic strips of finite width. Comparison of the numerical results with the analytics yields parameter values that had been unspecified by the analytics, and determines the modifications needed to describe the magnetization both near the strip center and near the boundaries. With no uniaxial anisotropy, the domain wall center can be described by the same hyperbolic secant form as with uniaxial anisotropy, but the effective anisotropy constant must now be thought of as increasing with increasing film thickness and decreasing with increasing film width.
\end{abstract}

DOI: 10.1103/PhysRevB.77.214419

PACS number(s): 75.60.Ch, 75.70.Kw

\section{INTRODUCTION}

Domain walls in ferromagnetic thin films have been a subject of extensive research for the last 70 years. ${ }^{1}$ Among many types of domain walls in thin ferromagnetic films, two are of particular interest to both theoreticians and experimentalists-the Néel wall (which, in the absence of perpendicular surface anisotropy, applies to the very thinnest films) and the Bloch wall. For the Néel wall, ${ }^{2,3}$ where the magnetization lies in-plane, the initial descriptions of the magnetization distribution included only exchange and uniaxial anisotropy. The first attempt to treat the dipoledipole interaction considered it as a perturbation ${ }^{4}$; however, the results were inconsistent with numerical simulations, ${ }^{5}$ which revealed that the domain wall exhibits a logarithmic falloff of magnetization far away from domain wall center. Later, again for systems with exchange, uniaxial anisotropy, and dipolar interaction, it was shown ${ }^{6-8}$ that such logarithmic behavior, originating from the presence of long-range dipoledipole interaction, can be obtained analytically by minimizing the domain wall energy, demonstrated in more detail in Refs. 9 and 10. A typical geometry, which will also be used in this work, is a Néel domain wall in a ferromagnetic strip that is infinite along $y$, has a half-width $w$ along $x$, and a thickness $h$ along $z$. The domain wall is centered in the middle of the strip $(x=0)$.

The present work extends the analysis for samples of finite width and thickness, both with and without uniaxial anisotropy and directly compares the results of an approximate analytical analysis and of our micromagnetic simulations, performed with the help of our micromagnetics package RKMAG. ${ }^{19}$ More specifically, we address the issue of both the asymptotic behavior of the domain wall far from the center as well as near the center. As a result we establish that an asymptotic analytical formula can be indeed correct far from the wall, but not too near the edge. Near the center one can simply assume that in addition to uniaxial anisotropy an effective shape anisotropy field is present, due to the dipoledipole interaction. For numerical calculations we will take material parameters appropriate to Permalloy-exchange stiffness $A=1.3 \times 10^{-6} \mathrm{erg} / \mathrm{cm}$, saturation magnetization $M_{s}$ $=795 \mathrm{emu} / \mathrm{cm}^{3}$, and zero anisotropy constant $K$, unless otherwise noted.

\section{ANALYTICS}

Let us first consider the problem of the Néel domain wall in an infinite thin ferromagnetic film lying in the $x y$ plane in the presence of exchange, the dipole-dipole interaction, and easy uniaxial crystalline anisotropy along $y$. Let the sample thickness $h$ be much smaller than both the exchange length $l_{e}$ and the anisotropy length $l_{a}$,

$$
l_{e}=\sqrt{\frac{A}{2 \pi M_{s}^{2}}}, \quad l_{a}=\sqrt{\frac{A}{K}},
$$

for permalloy $l_{e}=5.723 \mathrm{~nm}$ and $l_{a}$ is very large. (Shortly we will consider an effective anisotropy due to the short-range part of the dipole-dipole interaction.) Then the magnetization of the sample lies entirely in-plane, due to the dipole-dipole interaction, and is approximately uniform along $v(x)$ $=M_{y}(x) / M_{s}$. Therefore the magnetization $\vec{M}$ in the film can be described by the two dimensionless functions $u(x)$ $=M_{x}(x) / M_{s}$ and $v(x)=M_{y}(x) / M_{s}$, with $u(x)^{2}+v(x)^{2}=1$.

To obtain the magnetization distribution one minimizes the magnetostatic energy functional with the boundary conditions $v( \pm \infty)= \pm 1, u(0)=1$. The first condition specifies a magnetization reversal across the domain wall, and the second condition centers domain wall at $x=0$. The energy functional ${ }^{11}$ in the limit of a thin sample, with the account of translation symmetry along the $y$ axis, can be simplified to ${ }^{10}$

$$
\begin{aligned}
E[u]= & \frac{A h}{2} \int_{-\infty}^{+\infty}\left[\left(\frac{d u(x)}{d x}\right)^{2}+\left(\frac{d v(x)}{d x}\right)^{2}\right] d x \\
& -\frac{h^{2} M_{s}^{2}}{4} \int_{-\infty}^{+\infty} \int_{-\infty}^{+\infty}\left(\frac{1}{\left(x-x^{\prime}-i 0\right)^{2}}\right. \\
& \left.+\frac{1}{\left(x-x^{\prime}+i 0\right)^{2}}\right) u(x) u\left(x^{\prime}\right) d x d x^{\prime}+K h \int_{-\infty}^{+\infty} u(x)^{2} d x,
\end{aligned}
$$

where $K$ is the anisotropy constant. In this equation the first term is due to exchange, the second term is due to the longrange part of the dipole-dipole interaction, and the last term is due to in-plane uniaxial (crystalline) anisotropy in the direction perpendicular to the domain wall. 
In this section we are interested in the "tail" region outside the domain wall center, where $|v(x)| \approx 1$ and $[d v(x) / d x]^{2} \ll[d u(x) / d x]^{2}$. Thus ${ }^{10}$

$$
\begin{aligned}
E[u] \approx & \frac{A h}{2} \int_{-\infty}^{+\infty}\left(\frac{d u(x)}{d x}\right)^{2} d x-h^{2} M_{s}^{2} \int_{-\infty}^{+\infty} \int_{-\infty}^{+\infty}\left(\frac{1}{\left(x-x^{\prime}-i 0\right)^{2}}\right. \\
& \left.+\frac{1}{\left(x-x^{\prime}+i 0\right)^{2}}\right) \frac{u(x) u\left(x^{\prime}\right)}{4} d x d x^{\prime}+K h \int_{-\infty}^{+\infty} u(x)^{2} d x .
\end{aligned}
$$

The magnetization distribution, which minimizes the energy functional, can be obtained using the variational method, subject to the constraint that $M_{x}(0)=M_{s}$. Because our model does not describe the central part of the wall accurately, direct application of this constraint will give an incorrect normalization to $u(x)$. Therefore we introduce the parameter $\xi$ $=u(0)$, which will be obtained from the best fit of analytic solution to numerical data, or from matching the analytic solutions for the central and the tail regions. To deal with normalization, we therefore introduce a Lagrange multiplier $\Lambda$ via

$$
\begin{aligned}
\delta E[u]= & \int_{-\infty}^{+\infty}\left[-A \frac{d^{2} u(x)}{d x^{2}}-\frac{h M_{s}^{2}}{2} \int_{-\infty}^{+\infty}\left(\frac{1}{\left(x-x^{\prime}-i 0\right)^{2}}\right.\right. \\
& \left.\left.+\frac{1}{\left(x-x^{\prime}+i 0\right)^{2}}\right) u\left(x^{\prime}\right) d x^{\prime}+K u(x)\right] \delta u(x) d x \\
& -h M_{s}^{2} \Lambda \delta u(0) .
\end{aligned}
$$

At the energy minimum $\delta E[u]=0$ for any small $\delta u(x)$. Thus we obtain the equation for the magnetization distribution as follows:

$$
\begin{aligned}
& -A \frac{d^{2} u(x)}{d x^{2}}-\frac{h M_{s}^{2}}{2} \int_{-\infty}^{+\infty}\left(\frac{1}{\left(x-x^{\prime}-i 0\right)^{2}}\right. \\
& \left.+\frac{1}{\left(x-x^{\prime}+i 0\right)^{2}}\right) u\left(x^{\prime}\right) d x^{\prime}+K u(x)=h M_{s}^{2} \Lambda \delta(x) .
\end{aligned}
$$

Here we employ the Dirac delta function $\delta(x)$. Equation (2.4) greatly simplifies in the Fourier representation, with $\widetilde{u}(q)$ $=\int_{-\infty}^{+\infty} u(x) e^{i q x} d x$,

$$
\left(\frac{2 l_{\mathrm{ex}}^{2}}{h} q^{2}+|q|+\frac{2 l_{\mathrm{ex}}^{2}}{h l_{a}^{2}}\right) \tilde{u}(q)=\frac{\Lambda}{\pi} .
$$

The solution in the coordinate representation,

$$
u(x)=\frac{\Lambda}{\pi^{2}} \int_{0}^{\infty} \frac{\cos (q x)}{\varepsilon l_{a} q^{2}+|q|+\varepsilon / l_{a}} d q, \quad \varepsilon=\frac{l_{\mathrm{ex}}^{2}}{l_{a} h},
$$

where $\varepsilon$ is a dimensionless parameter. (For our permalloy calculations, $\varepsilon$ is determined by dipole-induced shape anisotropy, and is on the order of 0.1.) This integral can be expressed in terms of sine and cosine integrals.

For now, we are interested only in the tail of the domain wall. The corresponding values of $x$ are of the order of $l_{a}$, so the values of $q$ that provide the main contribution to the integral (2.6) are of the order of $\varepsilon / l_{a}$. Performing the integration for $\varepsilon \ll 1$ one obtains a very good approximation for $u(x)$ as follows:

$$
u(x)=\frac{\Lambda}{\pi^{2}}\left[\cos \left(\varepsilon \frac{x}{l_{a}}\right) \operatorname{ci}\left(\varepsilon \frac{x}{l_{a}}\right)+\sin \left(\varepsilon \frac{x}{l_{a}}\right) \operatorname{si}\left(\varepsilon \frac{x}{l_{a}}\right)\right],
$$

where $\operatorname{ci}(x)=\int_{x}^{\infty}(\cos t / t) d t$ is the cosine integral, and $\operatorname{si}(x)$ $=\int_{x}^{\infty}(\sin t / t) d t$ is the sine integral.

Let us discuss the asymptotic behavior of the solution. For large $x / l_{a}$ (the "far tail"), Kronmüller found that $u(x)$ can be approximated by power law dependence, ${ }^{12}$ or as follows in this case from Eq. (2.7),

$$
u(x \rightarrow \infty) \approx \frac{\Lambda}{\pi^{2} \varepsilon^{2}} \frac{l_{a}^{2}}{x^{2}} .
$$

For $x<l_{a}$ (the "near tail"), Eq. (2.6) can be approximated as

$$
u(x) \approx \frac{\Lambda}{\pi^{2}}\left[\text { const }-\ln \left(\frac{\varepsilon}{2} \frac{x}{l_{a}}\right)\right] .
$$

Thus in the near tail, by Eq. (2.9) $M_{x}$ should display a logarithmic decay as $x$ increases, and in the far tail by Eq. (2.8), $M_{x}$ should display an inverse square dependence on $x$.

In a ferromagnetic strip of finite width the integral (2.6) should have a cutoff for small $q$. For large enough $l_{a}$ the last term in the denominator of Eq. (2.6) plays exactly the same role as the proposed cutoff. Therefore we propose that the sample size can be accounted for by renormalizing $l_{a}$. In the absence of crystalline anisotropy $l_{a}$ should approximately equal the half width $w$. The presence of crystalline anisotropy should decrease $l_{a}$. We propose therefore a simple phenomenological equation for the renormalized anisotropy length $l^{*}$ entering Eq. (2.6) in place of $l_{a}$,

$$
\frac{1}{l^{*}} \approx \frac{1}{w}+\frac{1}{l_{a}},
$$

where $w$ is the half width.

\section{MICROMAGNETICS}

Analytical theory gave us a number of important clues to the asymptotic behavior of the magnetization, but so far offered little indication of how it depends on the parameters of a finite width sample, much of such dependence being contained in the dimensionless parameter $\Lambda$. We attempt to gain an additional insight by comparing Eq. (2.6) with micromagnetic calculations performed using our RKMAG code. ${ }^{19}$ We take the sample to be infinite along $y$, with the other parameters within physically acceptable ranges: for the half width $w$ we chose values between 75 and $5000 \mathrm{~nm}$, and for the thickness $h$ we chose values between 3 and $40 \mathrm{~nm}$, which is below the critical thickness at which the Néel wall to Bloch wall transition occurs. As noted above we take material parameters appropriate to permalloy, unless otherwise noted. In each calculation we obtain the equilibrium configuration using the relaxation method, ${ }^{13}$ followed by a Lyapunov ${ }^{14}$ local stability analysis to confirm the convergence. Since we dis- 


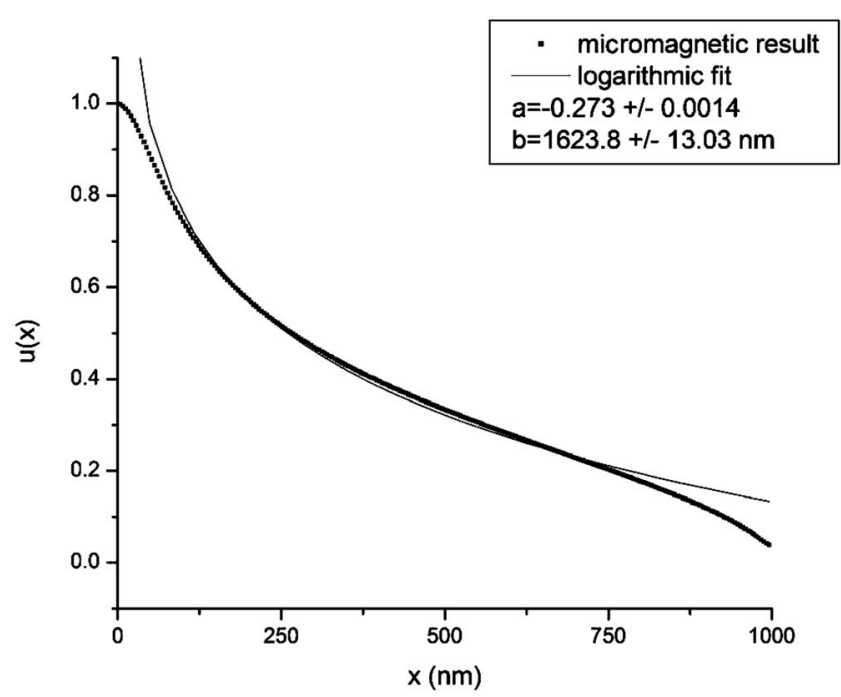

FIG. 1. Micromagnetic result and logarithmic fit $u(x)$ $=a \ln (x / b)$ for the following parameters: no anisotropy, $A=1.3$ $\times 10^{-6} \mathrm{erg} / \mathrm{cm}, M_{s}=795 \mathrm{emu} / \mathrm{cm}^{3}, w=1000 \mathrm{~nm}, h=5 \mathrm{~nm}$.

cretize the magnetic system by representing it as a large number of uniformly magnetized parallelepipeds, we test the discretization parameters for convergence by halving the parallelepiped size along any given direction. For the parameters above we obtain consistent, discretization-independent results for parallelepipeds that are $4 \mathrm{~nm}$ along $x, 4 \mathrm{~nm}$ along $y$, and no more than $7.5 \mathrm{~nm}$ along $z$. As often done in numerical micromagnetics, free boundary conditions are implemented at the sample's boundaries.

The first calculation neglects uniaxial anisotropy $(K=0)$, so that the domain wall is limited by the physical boundaries. The numerically obtained magnetization profile, given in Fig. 1, shows the presence of three distinctive regions, which we denote as "center", near tail, and "edge" (the region where boundaries play a significant role).

In the central region, which corresponds to $x<100 \mathrm{~nm}$ in Fig. 1, Eq. (2.6) overestimates $u(x)$. This is because for $K$ $=0$ the typical fitted values of $\Lambda$ are of order 0.2 , which is about $20 \%$ of the analytical prediction. This disagreement could have been anticipated since the assumption $u(x) \ll 1$ is clearly violated. Thus $\Lambda$ cannot be obtained from $u(0)=1$, but rather by stitching together the solutions in the "central" and tail regions.

We can modify the analytical approach by taking into account that in this case the domain wall formation is due to the competition of exchange and the effective shape anisotropy, Therefore we assume the form ${ }^{15,16}$ to be the same as in the anisotropy dominated case $u(x)=\sec h(x / \delta)$ (an example of a numerical fit is shown in Fig. 2), however, $\delta$ is related not to the uniaxial anisotropy length, but, $\delta \sim l_{e} \sqrt{1 / \beta}$, where effective shape anisotropy field $H_{\text {ani }}=\beta M_{y}$ and $\beta$ is a dimensionless function of the sample geometry. One can make a more precise estimate of $\beta$ by studying how the shape anisotropy field depends on $h$ and $w$. The magnetic field along $y$, produced in a uniformly magnetized magnetic prism that is infinite in the $y$ direction and averaged along the $x$-z plane, is given by ${ }^{17}$

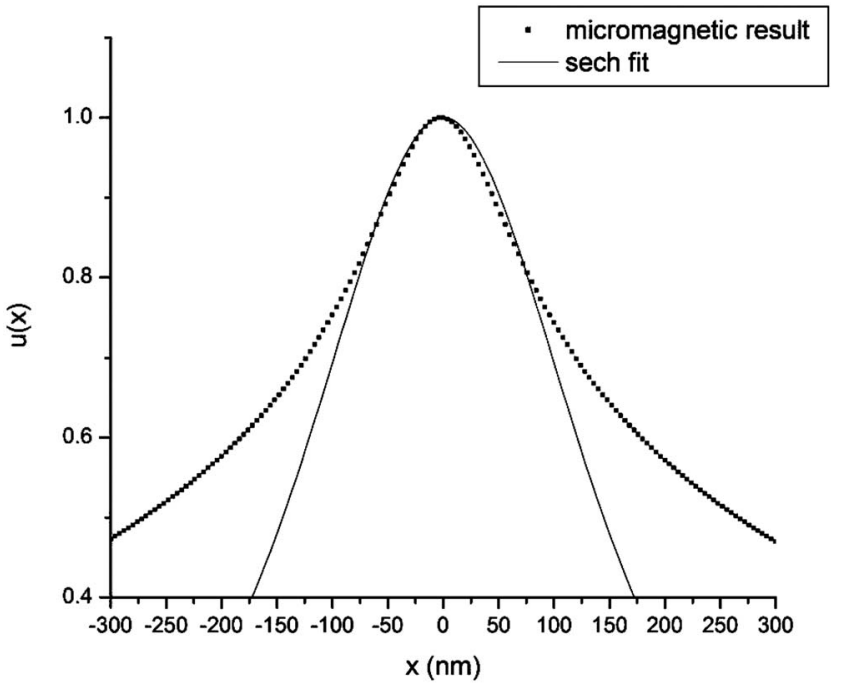

FIG. 2. Micromagnetic result and $u(x)=\sec h(x / \delta)$ fit, central portion of the domain wall, no anisotropy, $A=1.3 \times 10^{-6} \mathrm{erg} / \mathrm{cm}$, $M_{s}=795 \mathrm{emu} / \mathrm{cm}^{3}, w=1000 \mathrm{~nm}, h=5 \mathrm{~nm}, \delta=110 \mathrm{~nm}$.

$$
\beta \sim \frac{1-p^{2}}{2 p} \ln \left(1+p^{2}\right)+p \ln p+2 \arctan \left(\frac{1}{p}\right) p=\frac{h}{2 w} .
$$

Assuming that $h \ll w$, i.e., the sample is thin, we can expand $\beta$ as $\beta \approx \beta(0)+[d \beta(h) / d h] h$. Here $\beta(0)=$ const, the leading term in $d \beta(h) / d h$ is proportional to $1 / w$ and $\beta \sim h / w$, so that $\delta \sim l_{e} \sqrt{w / h}$.

Returning to Fig. 1 and $K=0$, we see that in the near tail region the magnetization profile falls off logarithmically, which is consistent with Eq. (2.9). The far tail region described by Eq. (2.8) does not occur. Thus, near the edge of the sample Eq. (2.6) predicts a logarithmic or slower falloff of the magnetization, whereas the numerics gives a falloff that is definitely faster than a logarithm of $x$. This is not surprising, since the formalism was developed for an infinite sample and nonzero uniaxial anisotropy, and thus neglected edge effects.

For nonzero $K$ the situation changes. If the anisotropy length is much smaller than the sample size, then edge effects are irrelevant. As shown in Fig. 3, the domain wall behavior outside of the central region can be very well described by Eq. (2.6), with the magnetization falloff as $1 / x^{2}$, rather than logarithmic, for $x>0.5 l^{*}$. The anisotropy then also defines the domain wall parameters $\delta$ and $l^{*}$.

Because the anisotropy-dominated regime has been studied extensively, ${ }^{15}$ we proceed to determine the contribution of the dipole-dipole interaction to domain wall parameters in the central and logarithmic tail regions. The sample has to be relatively wide or thin in order to observe the logarithmic asymptotics. Otherwise, if $l_{e}^{2} \approx w h$, then most of the magnetization profile will be exchange-dominated with $u(x)$ $=\sec h(x / \delta)$. With this knowledge we proceed to fit the numerically obtained $u(x)$, for different sets of parameters ( $w, h, A, M_{s}$ ), to the following function:

$$
u=\operatorname{sech}\left(\frac{\left(x-x_{0}\right)}{\delta}\right), \quad x<x_{c},
$$




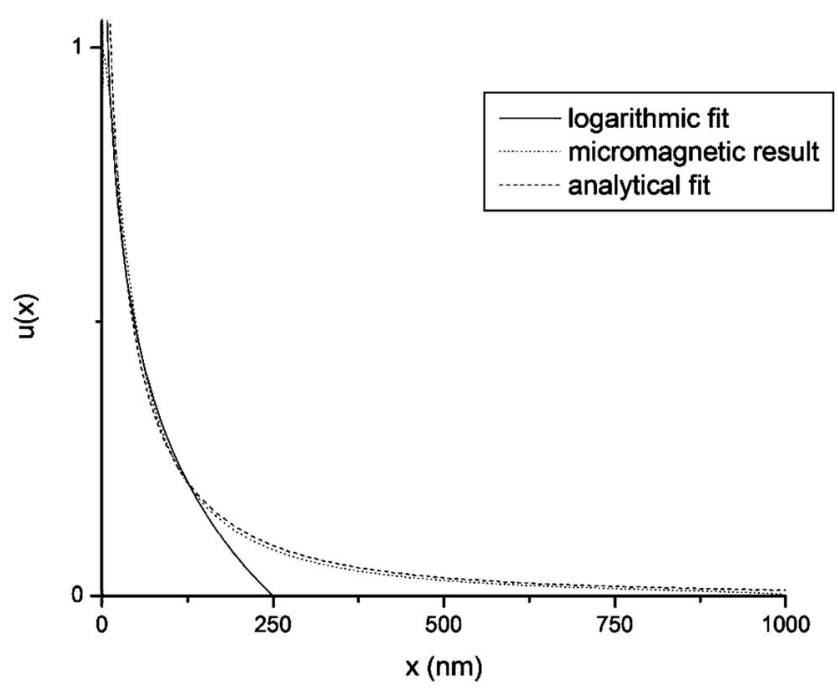

FIG. 3. Micromagnetic result, logarithmic fit of the logarithmic tail section $[u(x)=a \ln (x / b), a=-0.3, b=250 \mathrm{~nm}]$ and "analytical" $\left(l_{a}=150 \mathrm{~nm}, \Lambda=0.5\right)$ fit of the entire domain wall, $H_{\mathrm{ani}}=200 \mathrm{Oe}$, $A=1.3 \times 10^{-6} \mathrm{erg} / \mathrm{cm}, \quad M_{s}=795 \mathrm{emu} / \mathrm{cm}^{3}, \quad w=1000 \mathrm{~nm}, \quad h$ $=5 \mathrm{~nm}$.

$$
u=a \ln \left[\left(x-x_{0}\right) / l^{*}\right], \quad x_{c}<x<x_{e} .
$$

Here $a$ is the normalization constant, $l^{*}$ determines the speed of logarithmic falloff, $\delta$ is the domain wall center half width, $x_{0}$ is the position of the domain wall, and $x_{c}$ and $x_{e}$ define the region where the logarithmic approximation is valid. All these variables are fitting parameters. The following constraints are employed: $x_{c}$ must be relatively small since it separates the central and near tail regions; $x_{e}$ must be close to $w$, since $x_{e}$ separates the tail and edge regions; and $x_{0}$ should be 0 according to Eq. (2.6). A logarithmic dependence is used instead of Eq. (2.6) since, as was shown earlier, only in a logarithmic regime Eq. (2.6) is applicable since then edge effects can be neglected and $u \ll 1$.

We start with the following parameters: $A$ $=1.3 \cdot 10^{-6} \mathrm{erg} / \mathrm{cm}, M_{s}=795 \mathrm{emu} / \mathrm{cm}^{3}, h=12 \mathrm{~nm}$, and $w$ $=500 \mathrm{~nm}$. Then we successively adjust each of the parameters, while keeping the others fixed:

(a) $A$ is changed from $1.3 \times 10^{-6}$ to $3.6 \times 10^{-6}$ with a step size of $10^{-7} \mathrm{erg} / \mathrm{cm}$

(b) $h$ is changed from 3 to 38 with a step size of $0.5 \mathrm{~nm}$.

(c) $M_{s}$ is varied from 795 to 525 , with a step size of $15 \mathrm{emu} / \mathrm{cm}^{3}$.

(d) $w$ is varied from 250 to 1350 , with a step size of 50 nm.

The resulting equilibrium magnetization is then fitted using Eq. (3.2). As a result, the behavior of $\delta, a$, and $l^{*}$ can be analyzed. Unfortunately, since the number of fitting parameters is relatively large, such methods as Levenberg-Marquardt ${ }^{18}$ fail to lead to a global minimum. Moreover, because there is only a relatively small difference in quality of fit between the global and local minima, numerical uncertainty in the equilibrium configuration can easily cause the global and local minima to interchange. To sort this out, we employed a Monte Carlo method and obtained a

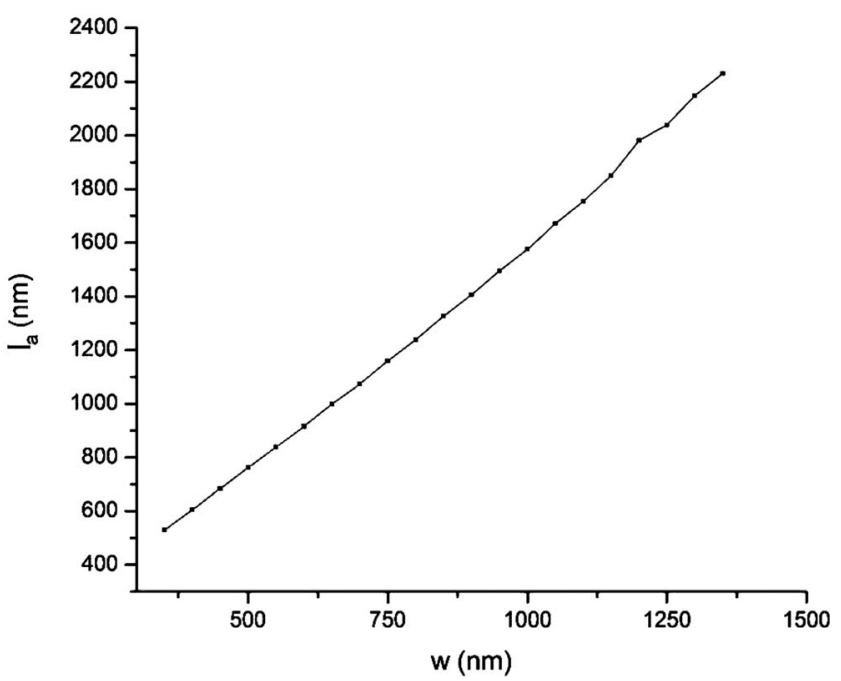

FIG. 4. Fitted value of $l_{a}$ (logarithmic fit) as a function of $w$, $A=1.3 \times 10^{-6} \mathrm{erg} / \mathrm{cm}, M_{s}=795 \mathrm{emu} / \mathrm{cm}^{3}, h=12 \mathrm{~nm}$.

number of local minima. Grouping them together shows the relative fitting error. For example, Fig. 4 shows a linear dependence of $l^{*}$ on $w$. Since the global minimum gives a significantly better correlation between the data and the fitting function than other minima, we can form a conclusion based solely on this graph.

Figure 5 shows the dependence of $a$ on $l_{e}$. There are a number of minima with similar correlations between the fit and the given data set; by performing numerous fittings we conclude that despite the large fitting error of about $14 \%$, the fitting parameters tend to group together and yield a linear dependence. In another case, shown in Fig. 6, a $5 \%$ or so lower rms between the data and the fit can be obtained if $c$ $=-0.42$ in $\delta=\delta_{0}+a h^{c}$. However, $c=-0.5$ makes more sense from a physical point of view, since $c=-0.42$, unlike

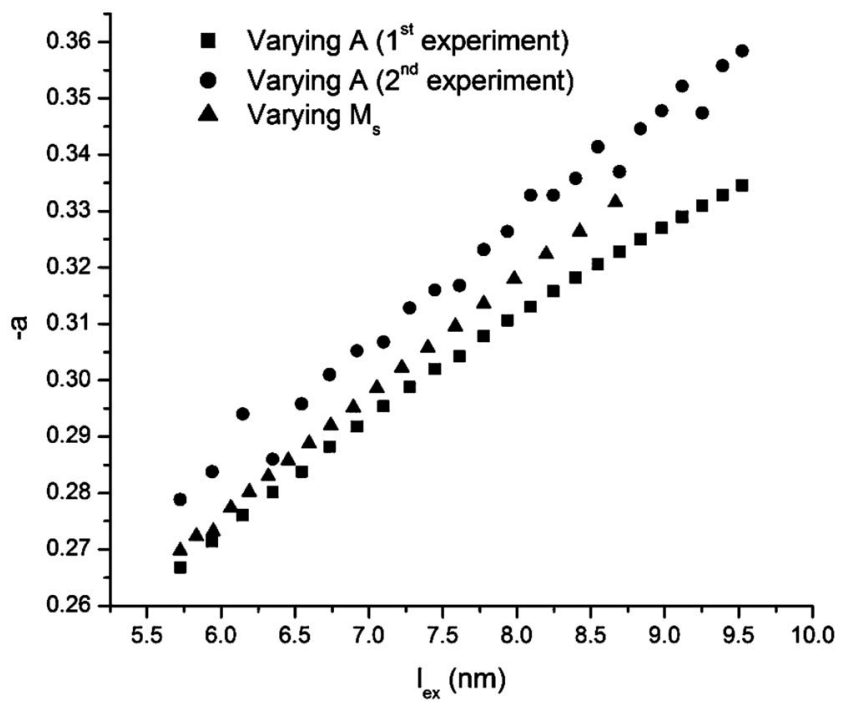

FIG. 5. Fitted value of a logarithmic fit as a function of $l_{\mathrm{ex}}, h$ $=12 \mathrm{~nm}, w=500 \mathrm{~nm}$. $A$ is changed from $1.3 \times 10^{-6}$ to $3.6 \times 10^{-6}$ with a step $10^{-7} \mathrm{erg} / \mathrm{cm}$, experiment repeated twice with different values corresponding to different local minima. Alternatively, $M_{S}$ is varied from 795 to 525 , step size $15 \mathrm{emu} / \mathrm{cm}^{3}$. 


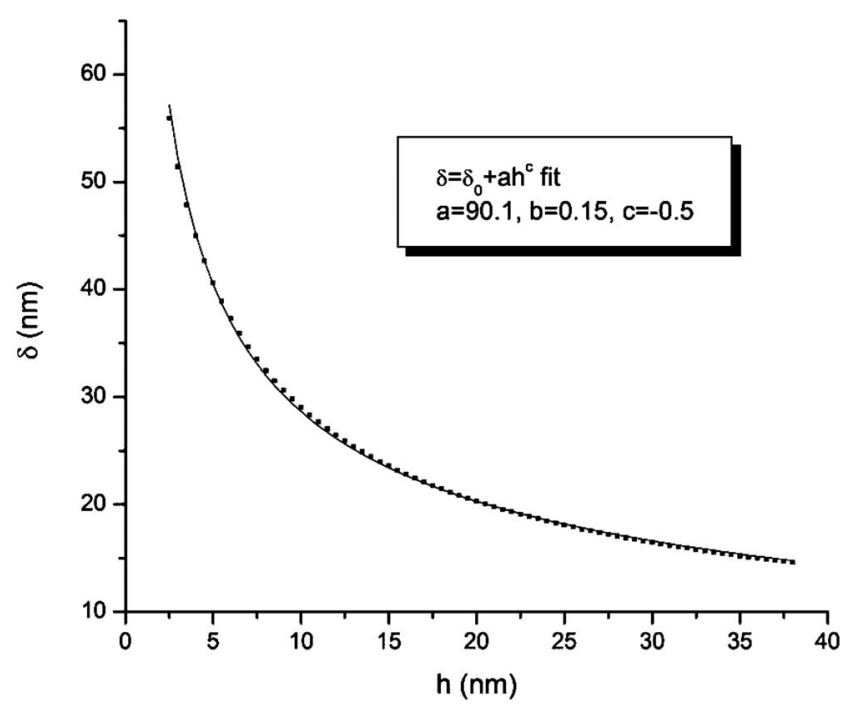

FIG. 6. $\quad w=100 \mathrm{~nm}, \quad A=1.3 \times 10^{-6} \mathrm{erg} / \mathrm{cm}, \quad M_{s}=795$ $\mathrm{emu} / \mathrm{cm}^{3}, \delta$ as a function of $h$.

$c=-0.5$, would require the presence of significant nonzero $\delta_{0}$. However, the fitting error for $c=-0.5$ is obviously large.

As a result of the numerical computations we were able to determine the following dependences:

$$
\begin{gathered}
u \sim \sec h\left(\sqrt{\frac{h}{w}} \frac{x}{l_{e}}\right), \quad x<x_{c}, \\
u \sim \frac{l_{e}}{\sqrt{w h}} \ln \left(\frac{x}{w}\right), \quad x_{c}<x .
\end{gathered}
$$

Thus $x_{0}$ is approximately 0 , as required by the sample's symmetry. Expression for $x_{c}$ can be obtained by stitching the above two solutions together.
We see that $\delta \sim l_{e} \sqrt{w / h}$, which agrees well with our analytical interpretation of the dipole field as a shape anisotropy field in this region. We find that in Eq. (2.9) one can indeed employ the effective anisotropy length $l^{*} \sim w$ and a comparison to the numerical results reveals the values of dimensionless constants $\Lambda=-\pi^{2} \sqrt{e}, C=\ln (\varepsilon / 2)$.

\section{SUMMARY}

Using both analytic and numerical methods we have studied the behavior of Néel walls in thin ferromagnetic strips subject both to exchange and the dipolar interaction with negligible uniaxial anisotropy. This problem is of interest because of the unusual logarithmic long-range tails that occur, and because it is relevant to permalloy.

Our studies establish that

(a) Equation (2.5) describes the magnetization profile of the Néel domain wall sufficiently far away from the domain wall center. When the anisotropy length $l_{a}$ is significantly greater than the sample width $w$, then $w$ should be used in place of $l_{a}$. Although we were not able to analytically identify the coefficients involved in Eq. (2.10), we have shown numerically that it corresponds to a logarithmic dependence of the form $u \sim\left(l_{e} / \sqrt{w h}\right) \ln (x / w)$, except for a small region near the boundary where edge effects cannot be neglected.

(b) In the domain wall center the same formula applies for both dipole-dipole and anisotropy-dominated domain walls. However, for the dipole-dipole dominated case, the shape anisotropy field replaces the uniaxial anisotropy field and $\delta$ $\approx l_{e} \sqrt{w / h}$.

\section{ACKNOWLEDGMENT}

This work was sponsored by DOE Grant No. DE-FG0206ER46278.
${ }^{1}$ F. Bitter, Phys. Rev. 38, 1903 (1931).

${ }^{2}$ L. Neel, C. R. Hebd. Seances Acad. Sci. 241, 533 (1955).

${ }^{3}$ S. Middelhoek, IBM J. Res. Dev. 10, 351 (1966).

${ }^{4}$ R. Collette, J. Appl. Phys. 35, 3294 (1964).

${ }^{5}$ R. Kirchner and W. Doring, J. Appl. Phys. 39, 855 (1968).

${ }^{6}$ H. Riedel and A. Seeger, Phys. Status Solidi B 46, 377 (1971).

${ }^{7}$ A. Hubert, IEEE Trans. Magn. 11, 1285 (1975).

${ }^{8}$ A. Hubert, Theorie der Domanenwande in geordneten Medien, Lecture Notes in Physics Vol. 26 (Springer-Verlag, Berlin, 1974).

${ }^{9}$ C. Garcia-Cervera, Eur. J. Appl. Math. 15, 451 (2004).

${ }^{10}$ C. Melcher, Arch. Ration. Mech. Anal. 2, 168 (2003).

${ }^{11}$ A. Aharoni, Introduction to the Theory of Ferromagnetism (Oxford University Press, Oxford, 1996).

${ }^{12}$ H. Kronmüller, Phys. Status Solidi 11, K125 (1965).

${ }^{13}$ K. Rivkin, A. Heifetz, P. R. Sievert, and J. B. Ketterson, Phys.
Rev. B 70, 184410 (2004).

${ }^{14}$ Emil Simiu, Chaotic Transitions in Deterministic and Stochastic Dynamical Systems (Princeton University Press, Princeton, NJ, 2002).

${ }^{15}$ L. Landau, E. Lifshitz, and L. Pitaevskii, Course of Theoretical Physics (Pergamon Press, Oxford, 1985), Vol. 8.

${ }^{16}$ Radu Ignat and Felix Otto, SFB Preprint, 160, 2007 (http://www-mathphys.iam.uni-bonn.de/ otto/publications/ IgnatOtto.pdf). This work considers exchange, uniaxial anisotropy, the dipolar interaction, and a sample of infinite width.

${ }^{17}$ A. Aharoni, J. Appl. Phys. 83, 3432 (1998).

${ }^{18}$ H. William Press, Saul A. Teukolsky, William T. Vetterling, and Brian P. Flannery, Numerical Recipes in C, 2nd ed. (Cambridge University Press, Cambridge, England, 1992), p. 393.

${ }^{19}$ www.rkmag.com 